\title{
Noise-Assisted Multivariate Empirical Mode Decomposition Based Emotion Recognition
}

\author{
Pınar Özel', 4 (D), Aydın Akan² (D), Bülent Yılmaz \\ 'Department of Electrical and Electronics Engineering, Nevşehir Hacı Bektaş Veli University School of Engineering, Nevşehir, Turkey \\ 2Department of Biomedical Engineering, İzmir Katip Celebi University School of Engineering, Izmir, Turkey \\ ${ }^{3}$ Department of Electrical and Electronics Engineering, Abdullah Gul University School of Engineering, Kayseri, Turkey \\ ${ }^{4}$ Biomedical Engineering Program, Institute of Science, İstanbul University, İstanbul, Turkey
}

Cite this article as: P. Özel, A. Akan, B. Yılmaz, "Noise-Assisted Multivariate Empirical Mode Decomposition Based Emotion Recognition", Electrica, vol. 18, no: 2, pp. 263-274, 2018.

\begin{abstract}
Emotion state detection or emotion recognition cuts across different disciplines because of the many parameters that embrace the brain's complex neural structure, signal processing methods, and pattern recognition algorithms. Currently, in addition to classical time-frequency methods, emotional state data have been processed via data-driven methods such as empirical mode decomposition (EMD). Despite its various benefits, EMD has several drawbacks: it is intended for univariate data; it is prone to mode mixing; and the number of local extrema must be enough before the EMD process can begin. To overcome these problems, this study employs a multivariate EMD and its noise-assisted version in the emotional state classification of electroencephalogram signals.
\end{abstract}

Keywords: Emotion recognition, electroencephalography, empirical mode decomposition, multivariate empirical mode decomposition, noise assisted multivariate empirical mode decomposition

\section{Corresponding Author: \\ Pınar Özel}

\section{E-mail:}

pinarozel@nevsehir.edu.tr

Received: 02.03 .2018

Accepted: 10.05 .2018

(c) Copyright 2018 by Electrica

Available online at

http://electrica.istanbul.edu.tr

DOI: 10.26650/electrica.2018.00998

\section{Introduction}

Electroencephalography (EEG), introduced by Hans Berger in 1924, is a noninvasive method that utilizes electrical potential recordings on the scalp at different locations at microvolt level. A brain-computer interface $(\mathrm{BCl})$, an innovation based on computer-assisted controls utilizing brain activity, depends on EEG signals and provides a means to discover a variety of uses ranging from bioengineering to neuro-prosthetics. These new advancements in human-computer interaction applications also focalize the transfusion of emotional states regarding information between the brain and the computer. Thus, in the literature, there are numerous studies on emotional state modeling [1-3]. Yet, the most commonly used two-dimensional or three-dimensional space is a circumplex model that shows the emotional state as continuous points. In the two-dimensional space, emotions are displayed by arousal-valence map and with respect to three-dimensional space, they are modelled as arousal-valence-dominance (VAD) map. In these models of emotions, emotional states are one of the qualities of physiological-neural aspects of emotions, which are isolated from each other, and are represented as a blend of these dimensions. Arousal is defined as the power or intensity of sensation (emotional arousal), valence is defined as the satisfaction or dissatisfaction grade (emotional valence), and dominance is defining as the power of controlling emotion internally (emotional dominance). For example, anger is shown as a combination of negative valence and high arousal [4].

Traditional time-frequency representation algorithms, such as short-time Fourier transform (STFT) and continuous wavelet transform (CWT), have been utilized to frequently examine the emotional state data [5].Yet such methods restrict the representations in time-frequency space depending on the projection of data onto the fixed arrangement of the fundamental 
functions. Recently, improvements have been presented using several techniques for time-frequency analysis depending on the data itself.

The empirical mode decomposition (EMD) transforms the signal into an arrangement of band-limited segments leaving well-localized patterns at the instantaneous frequency level. There are no previous assumptions for the basic signal properties, which are adapted for nonlinear and nonstationary data analysis in EMD method. In other words, in contrast to fixed fundamental functions in Fourier and wavelet transforms, EMD expands a given time series $x(k)$ to a set of narrowband oscillation modes, called intrinsic mode functions (IMFs) naturally occurring from self-existing oscillations in the signal $x(k)$ [6]. It provides great superiority in executing real-world signals because it is data-adaptive, comprehensive, and much more flexible than Fourier and wavelet-based functions. The non-linearity inherent in the EMD algorithm also gives a compact representation possibility. Subsequently, normalized Hilbert Transforms, and quadratic methods can be used to acquire more precise time-frequency representations [7]. However, the operation of EMD has underestimated certain issues, such as specific mode mixing and overlapping.

In this paper, section 2 summarizes the related works about the proposed model. Section 3 explains the content of the data set and gives an explanation of the algorithms related to EMD, multivariate EMD (MEMD), and noise-assisted multivariate EMD (NA-MEMD). In section 4, we present a broad set of simulations and emotional state classification results for real emotional state data compared to NA-MEMD. Finally, in section 5 , the implementations of NA-MEMD to non-stationary EEG signals are outlined in discussion and conclusions.

\section{Related Work}

To date, EMD-based emotional recognition methods have been developed using standard single and multiple channel EEG signals [8-17]. In addition, the researchers whose studies are cited in performed emotional state recognition using multivariate EMD due to the idea of multiple channel approach [18-22]. Despite the advantages of the EMD method for linear and non-stationary data, it has the disadvantage that it is practically not arithmetical [6]. The susceptibility of the EMD algorithm to local signal changes can often bring about decompositions [23]. However, the process of the EMD method tends to cause "mode-mixing" and "aliasing" due to sub-Nyquist extreme sampling, which is often caused by overlaid IMF spectra. In order to overcome these disadvantages a new approach called noise-assisted multivariate empirical mode decomposition (NA-MEMD) has recently been developed [24]. As an important step in data-adaptive analysis, the applications of NA-MEMD have so far been utilized resulting in positive outcomes, which are based on time-frequency axes with Doppler radar signals computer simulations and motor image EEG data from the $\mathrm{BCl}$ competition IV data set in time-frequency analysis of neuronal populations with instantaneous resolution phase synchronization using EEG-based prediction of epileptic seizures, in lung-heart sound discrimination, multichannel EMG signals, and rejecting the unwanted noise contained within the VLF-EM (very low-frequency electromagnetic method) data, which produced NA-MEMD [25-31]. Additionally, as an improved noise assisted method for multivariate signals decomposition, partial noise assisted multivariate EMD is proposed by Huang et al. [32].

In this study on emotional state analysis, NA-MEMD is evaluated as a potential method for the processing of EEG signals and the results are compared with MEMD and EMD methods. That is, we present the applications of NA-MEMD, as well as the algorithm of EMD, which seems not enough for multivariate time series, and MEMD contribution as its multivariate extension. We will relate the decomposition by emphasizing the contribution of the IMF differences via the noise subspace added to the input data to demonstrate the mode-alignment and filter bank properties of NA-MEMD.

\section{Methodology}

\section{Dataset}

The DEAP database was utilized in this investigation which consists of the dimensional features-valence, arousal, and dominance- to depict distinctive emotional diversity for 32 participants [33]. While creating the database the subjects first viewed 120 videos and 40 of them were selected as suitable stimulants because they displayed the most stable emotions with reference to the cognitive affective map. The ratio to the standard deviation of the averages of the values given for each video was fixed for the experiment credibility indicator. During the video viewing 32-channel EEG data were acquired. Thus, our data set consisted of 32-channel EEG data and the corresponding questionnaire answers using self-assessment mannequin (SAM) that showed the participant's responses to valence, arousal and dominance.

\section{Empirical Mode Decomposition}

EMD is a completely data-driven method that achieves highly localized time-frequency estimation for linear and non-stationary signals. Thus, the signal is converted into a finite set of IMFs, which are amplitude and frequency modulated (AM and FM) waves. Two requirements are essential for a signal to be regarded as an IMF.

1) The number of extrema and the number of zero crossings should be different only by one.

2) The average envelope that connects the local maxima and minima, respectively, is roughly zero.

Therefore, as an advantage of EMD compared to Fourier techniques, each IMF can be treated as a narrow-band signal externalizing a different time-scale specific to the data set CITATION 
Nor98 \ 1055 [6](Norden E. Huang, 1998). EMD process is summarized below:

\section{Algorithm 1: Standard EMD algorithm}

1) Assume that $v(t)$ is the initial signal, let $\tilde{v}(t)=v(t)$

2) The local maximum and local minimum of $\tilde{v}(t)$ are found.

3) A lower 'envelope' exists as $e_{l}(t)$ that interpolates all local minima.

4) A higher 'envelope' is found as $e_{u}(t)$, which interpolates all local maxima.

5) As $\bar{m}(t)=\left(e_{l}(t)+e_{u}(t)\right) / 2$, the local average is calculated.

6) The local average is subtracted from $\tilde{v}(t), c_{i}(t)=\tilde{v}(t)-\bar{m}(t)$ (where $i$. is the first order of the IMF).

7)

$\tilde{v}(t)$ is assigned as $c_{i}(t)$, and it is passed to the second step after $c_{i}(t)$ is IMF.

The first IMF is removed from the initial data, $r(t)=v(t)-c_{1}(t)$ , and this is executed repeatedly until no more oscillations are present or it is fixed. This process, referred to as 'sifting process', is controlled appropriately by $a$ defined stopping criterion. The signal $v(t)$ is defined in equation 1 .

$v(t)=\sum_{i=1}^{M} c_{i}(t)+r(t)$

where $c_{i}(t), i=1, \ldots \ldots, M$ are IMFs and $r(t)$ is the residual. The narrowband structure of IMFs is suitable for the Hilbert Transform which can be operated to acquire localized time-frequency spectrograms.

$$
H\left(c_{i}(t)\right)=\frac{1}{\pi} P\left(\int_{-\infty}^{+\infty} \frac{c_{i}\left(t^{1}\right)}{t-t^{1}} d t^{1}\right.
$$

where the symbol $P$ denotes the Cauchy principal value and the analytical signal is obtained as:

$$
v(t)=\sum_{i=1}^{M}\left(c_{i}(t)+j H\left(c_{i}(t)\right)=\sum_{i=1}^{M} a_{j}(t) e^{j Q_{i}(t)}\right.
$$

And the amplitude and phase functions are defined as $a_{i}(t)$ and $Q_{i}(t)$ respectively. The phase function $Q_{i}(t)$ is used to derive the instantaneous frequency $w_{i}(t)=\frac{d Q_{i}(t)}{d t}$. The graph of the amplitude versus time, $\mathrm{t}$, and the instantaneous frequency $w_{i}(t)$ more precisely, the determination of the amplitude curve over the time frequency axis is known as the Hilbert-Huang Transform (HHT).

\section{Multivariate Empirical Mode Decomposition}

Multivariate EMD is the extension of the standard, bivariate and trivariate EMD developed by Rehman and Mandic [34]. The standard EMD calculates the local average applying the average of the up and down envelopes. Nevertheless, the local average of an $\mathrm{n}$-dimensional signal cannot be computed in a direct way, and multidimensional envelopes are obtained by projecting the signal in variable spaces along different directions, then averaging these reflections to attain the local average. For the set of direction vectors utilized to reflect the input of a multivariate signal, low discrepancy Hammersley sequences were exploited to make semi-uniform dots on high-dimensional spheres, giving uniformly distributed direction vectors $(\mathrm{V})$ on $\mathrm{n}$ spheres and making the resulting strategy precise and computationally effective [34].

Notwithstanding, the issue of selecting a reasonable arrangement of direction vectors for performing signal projections in $\mathrm{n}$-dimensional space necessitates a caution. The prediction of the local mean along multiple directions in an n-dimensional space can be viewed as a calculation of the integral of all the envelopes and the precision of calculation arises from the direction vectors being selected consistently, particularly for a restricted quantity of direction vectors. Because the direction vectors in $\mathrm{n}$-dimensional spaces may be identically symbolized as points on the relating unit $(n-1)$ spheres, the issue of selecting an appropriate arrangement of direction vectors may be treated as that of discovering a uniform sampling scheme on $\mathrm{n}$ sphere. Once an appropriate arrangement of direction vectors on the $n$ sphere is produced, projections of the signal are ascertained along that arrangement; the extremum of projected signals are interpolated to provide the required multidimensional envelopes of the signal. The average of multiple envelope curves, each having a special direction vector, is taken to get the multivariate signal mean.

The details of the MEMD are summarized below:

\section{Algorithm 2: Multivariate EMD algorithm (MEMD)}

1) An appropriate point for sampling over $(n-1)$ sphere is selected.

2) For all $k$ (all clusters of direction vectors), to give $\left\{p^{Q_{k}}(t)\right\}_{k=1}^{K}$ as a reflection set, $\left\{p^{Q_{k}}(t)\right\}_{t=1}^{T}$ is calculated by the reflection of input signal $\{v(t)\}_{t=1}^{T}$ along direction vector $x^{Q_{k}}$.

3) The time constant $t_{j}{ }^{Q_{k}}$ corresponding to the maximum of the reflected signals $\left\{p^{Q_{k}}(t)\right\}_{k=1}^{K}$ is found.

4) Interpolation is performed using $\left[t_{j}^{Q_{k}}, v\left(t_{j}^{Q_{k}}\right)\right]$ to obtain highly variable envelope curves $\left\{e^{Q_{k}}(t)\right\}_{k=1}^{K}$.

5) For a set of $K$ direction vectors, the average of the envelope curves is computed as $m(t)=\frac{1}{K} \sum_{k=1}^{K} e^{Q_{k}}(t)$

6) Where ' $i$ ' is the order of the IMF, 'detail' $c_{i}(t)$ is extracted utilizing, $c_{i}(t)=v(t)-m(t)$. If 'detail' $c_{i}(t)$ satisfies the stopping criterion for a multivariate IMF, the above procedure is applied to $v(t)-c_{i}(t)$, if not, it is applied to $c_{i}(t)$.

\section{Limitations of Single and Multichannel Data Analysis with EMD}

When the EMD is applied separately for each EEG channel, the IMFs acquired from different EEG channels are dissimilar in 
number and have distinct statistical characteristics due to the EEG signals problem of uniqueness. Moreover, the EMD is reflected by different decompositions acquired from the signals of similar statistics, and thus, mode-mixing phenomenon occurs in the EMD process at similar frequencies for different IMFs. In the MEMD, the first IMFs acquired from different EEG channels have parallel frequency fluctuations, bandwidths, or autocorrelation features. However, MEMD still exhibits an amount of mode-mixing sensitivity. Mode mixing occurs with a single IMF carrying signals of essentially different modes or a single mode in more than one IMF [18]. This is a consequence of the signal spacing that causes the IMF spectrum to intersect and overlap in the time-frequency domain. Two sources of overlapping are i) extremum sampling or sub-Nyquist sampling which is an important step in EMD filtering, and ii) the equivalent interpolation filter (e.g. a cubic spline) of the EMD away from the ideal low-pass filter creates more overlapping. It is important to emphasize that the EMD filtering process is strongly dependent on the sampling of the signal extremum points, and thus, the existence of a sufficient number of extrema in an input is a precondition for the initiation and successful execution of the EMD approach. For example, a standard EMD fails to work on a Dirac pulse because there are not enough extrema for an expressive EMD. To get an equivalent noise response from the EMD filter, Flandrin et al. [35] considered the limit of the noise pulse to be an idealized impulse pulse and executed the EMD.

The addition of noise to the Dirac pulse gives rise to additional extremum points for the signal that assists the commencement of the EMD filtering process and acquires its equivalent impulse response. The concept of adding a noise set into the signal forms the basis of ensemble empirical mode decomposition (EEMD) [36]. The EEMD works by adding many independent noise sets to the data and applies EMD to each member of the resulting ensemble. In doing so, the EEMD uses the dyadic filter bank feature of the EMD when applied to the white gaussian noise (WGN). In addition to the analysis without mode-mixing, EEMD reveals the more localized modes inherent in the data. However, because of adding noise in a direct way to the data, it is probable that residual noise effects remain in IMFs. The amplitude of these remaining depends on the number of implementations averaged over the ensemble size, risking the completeness of the remaining signal. Various changes in the EEMD have been recommended to overcome this issue. Torres et al. [37] worked on carefully selected noise at each analysis stage to calculate a single residue. Complementary ensemble empirical mode decomposition (CEEMD) removes residuals from the WGN through the complementary ensemble IMF pairs acquired from positive and negative sections of the added noise [38].

\section{Noise-Assisted Multivariate Empirical Mode Decomposition.}

Noise is normally a substance added in that it adds to the data bearing signal. A model of the received signal with additive noise is represented as:
Considering signal to noise ratio is given by:

$$
\frac{S}{N}=\frac{S . P}{N . P}
$$

Where $S$ stands for signal and $N$ stands for noise and also S.P and N.P are abbreviations of Signal and Noise Power. The signal to noise $(S N R)$ in $d B$ expressed by:

$$
\left(\frac{S}{N}\right)_{d B}=10 \log _{10}\left(\frac{S}{N}\right)
$$

Additive WGN utilized in this study is a basic and usually accepted noise model in signal processing and is the arrangement of assumptions that the noise samples have a Gaussian distribution and are white which means power spectral density is horizontally stable and hence the autocorrelation of the noise in time domain is zero for any non-zero time offset. Additionally, the noise is additive where the noise is statistically independent of the signal and the received signal equals the transmit signal plus some noise as can be seen in Figure 1.

Hence, the NA-MEMD first runs by forming a multivariate signal containing one or more input data channels and adjacent independent events of the WGN in separate channels. The multivariate signal, which consists of data and noise channels, is processed utilizing the MEMD method and the IMFs corresponding to the initial data are reconstituted to obtain the desired decomposition. In this way, unlike the EEMD, physically separate input and noise subspaces in the NA-MEMD prevent direct noise artifacts. In the multivariate data shifting process, when all reflected signals meet any stopping criteria accepted in the standard EMD, IMF can be stopped. The MEMD algorithm, when applied by adding WGN to a multidimensional signal, acts as a dyadic filter bank on each channel, showing a greatly improved rank order of IMFs corresponding to different channels over the same frequency range when compared to EMD as can be seen in Figures 2, 3. Using this feature of MEMD, Rehman and Mandic [34] proposed the noise assisted MEMD method to further improve the mixing problem CITATION Nav13 \ 1055 [24]. This is accomplished by including a subspace with multivariate independent WGN and increasing the size of the data, and the resulting composite signal is processed using MEMD. In this way, the noise will remain in a different

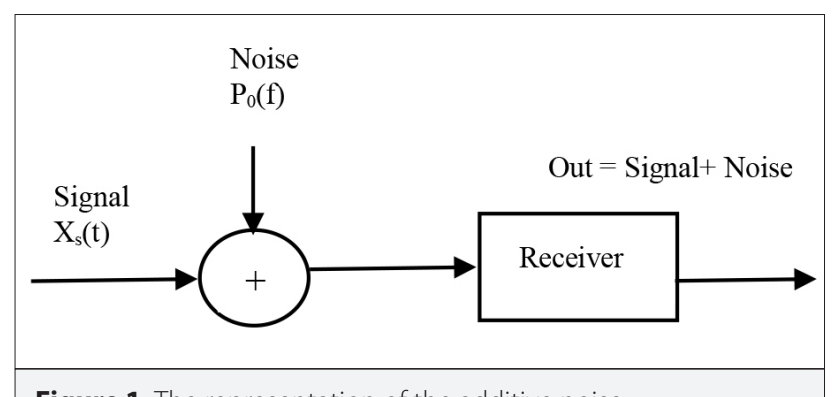

Figure 1. The representation of the additive noise 


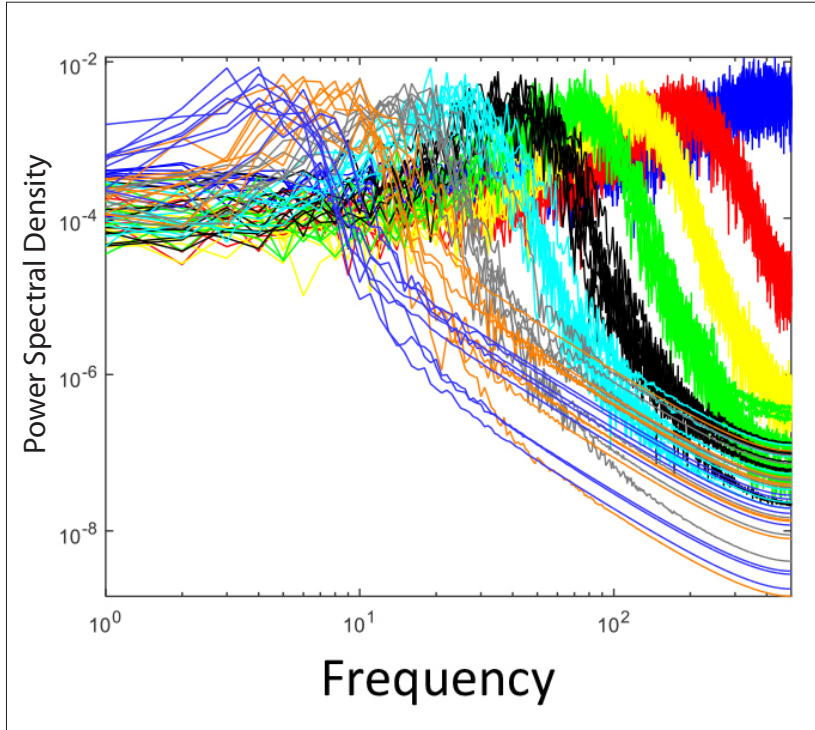

Figure 2. Mod mixing demonstration on IMFs using EMD as a filter bank

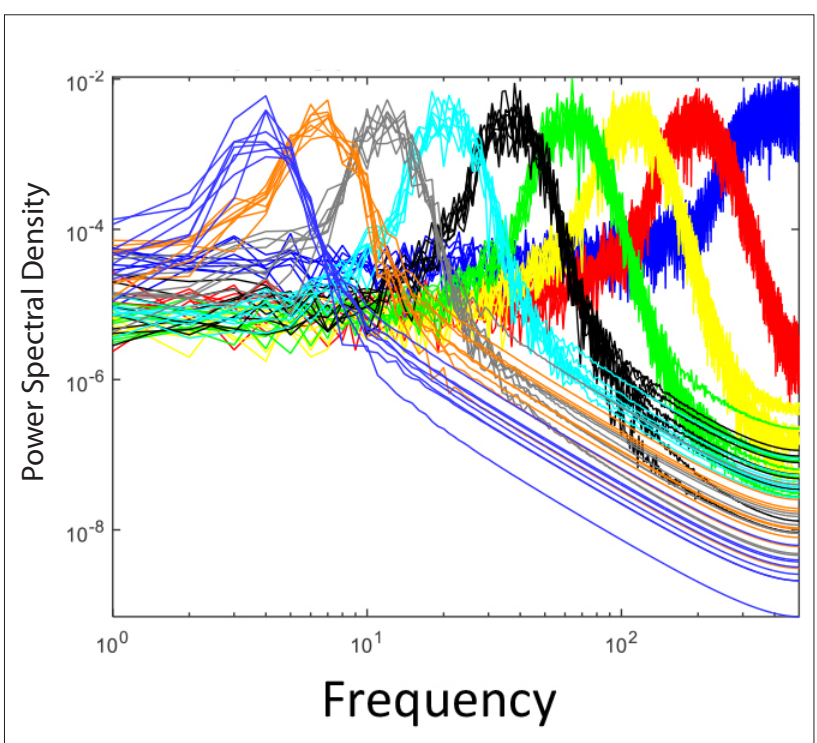

Figure 3. Mod mixing demonstration on IMFs using MEMD as a filter bank

subspace and be used in raising the filter bank structure, never interfering with the useful data channels, thereby reducing the issue of mode-mixing and providing a better definition of the frequency bands. Only the IMFs corresponding to the initial input signal are preserved by subtracting the noise-dependent IMF subspace. Due to the noise subspace, the alignment of the IMFs adapts to the dyadic filter bank structure, thus providing an important tool for non-stationary analysis of narrow band biomedical signals and aligning the IMFs related to the original input signal. Regardless of the differentiation between the EEMD and NA-MEMD, the main reason behind both methods is to use the dyadic filter bank structure of the (M) EMD, both
EMD and MEMD approaches, by adding the WGN to the signal for reformed efficiency of the standard EMD. The NA-MEMD algorithm's output depends on the power level of the attached noise channels. The algorithm performs like the standard EMD for infinite small noise amplitudes. Enhancing the power of noise will further strengthen the structure of the dyadic filter bank on the input data, but it overrides the data adaptive capability of the (M) EMD-based algorithms. The ideal noise levels for NA-MEMD are within $2-10 \%$ of the input data power (variance) [24].

\section{Algorithm 3: Noise Assisted Multivariate EMD}

1) Create a series of uncorreleted white Gaussian noises with the same length as the input ( $q$ channels).

2) Add the noise channel ( $q$ channel) obtained in step 1 apart from multivariable signal ( $n$ channel) as an input, and obtain a signal of $(n+q)$ channel.

3) Using the MEMD algorithm summarized in Algorithm 2, the resulting $(n+q)$ channel multivariate signal is processed.

4) The resulting $(n+q)$ variable IMF, the $q$ channel corresponding to the noise is removed, thus, the cluster of IMFs corresponding to the original signal as $n$ channel remains.

Recently, different methods have also been proposed in which the assistant signal for the input signal (a fractional gaussian noise and sinusoidal signal apart from WGN are utilized [39-41]. The details of the NA-MEMD are as Figure 2 and Figure 3.

\section{Proposed Methods}

\section{Mod Mixing Definition and Demonstration}

Considering signal $x(n)$, the MEMD and NA-MEMD algorithms were applied to the emotional EEG signal, yielding the $\operatorname{IMF}_{\mathrm{k}}(\mathrm{n})$ for modes $k=1, \ldots ., K$ for MEMD and the $\mathrm{IMF}_{1}(\mathrm{n})$ for modes $l=1, \ldots . ., L$, for NA-MEMD, mod mixing problem is demonstrated below:

$I M F_{k, l}(n)_{M o d-M i x i n g}=\sum_{k=1}^{K} I M F_{k}(n)-\sum_{l=1}^{L} I M F_{L}(n)$

Accordingly, the EEG signals taken from the $10^{\text {th }}$ participant's $20^{\text {th }}$ video in DEAP dataset expanded to their IMFs utilizing MEMD is presented in Figure 4 and the expansion of the same EEG signal to IMFs using NA-MEMD is presented in Figure 5. In total, the first 10 IMFs, which seemed to be meaningful, were presented visually. It seemed as if there are no differences between the expansion of IMFs oscillations between MEMD and NA-MEMD, presented in Figure 4, 5. However, to better understand the issue between IMF expansions obtained using these methods, the differences (having been acquired by subtracting IMFs oscillations of NA-MEMD (Figure 5) from IMFs oscillations of MEMD (Figure 4) were taken into consideration and Figure 6 is presented. 


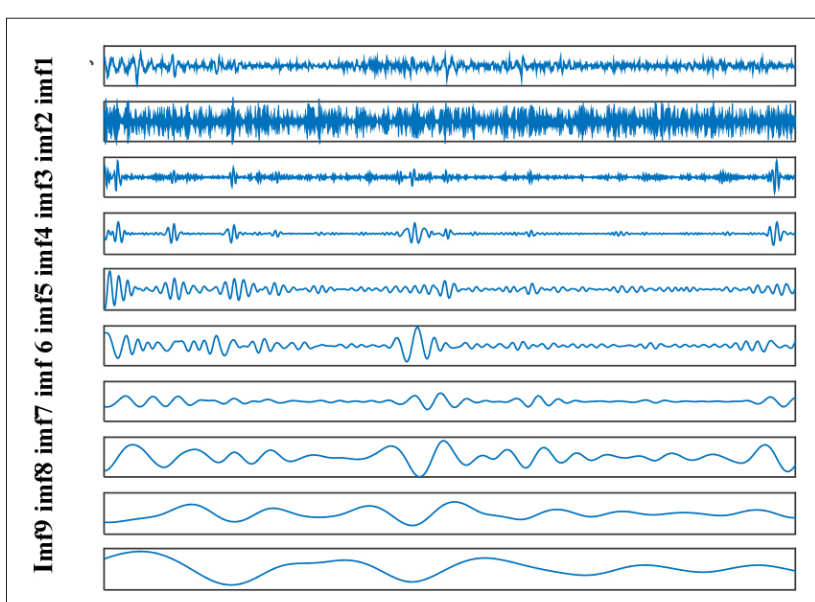

Figure 4. Expansions of EEG signal to IMF oscillations, using MEMD

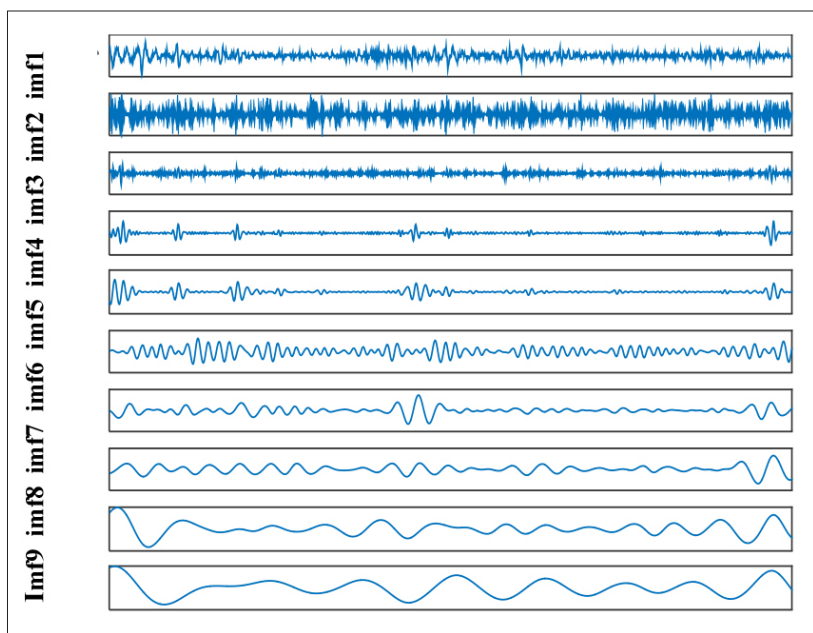

Figure 5. Expansions of EEG signal to IMF oscillations, using NAMEMD

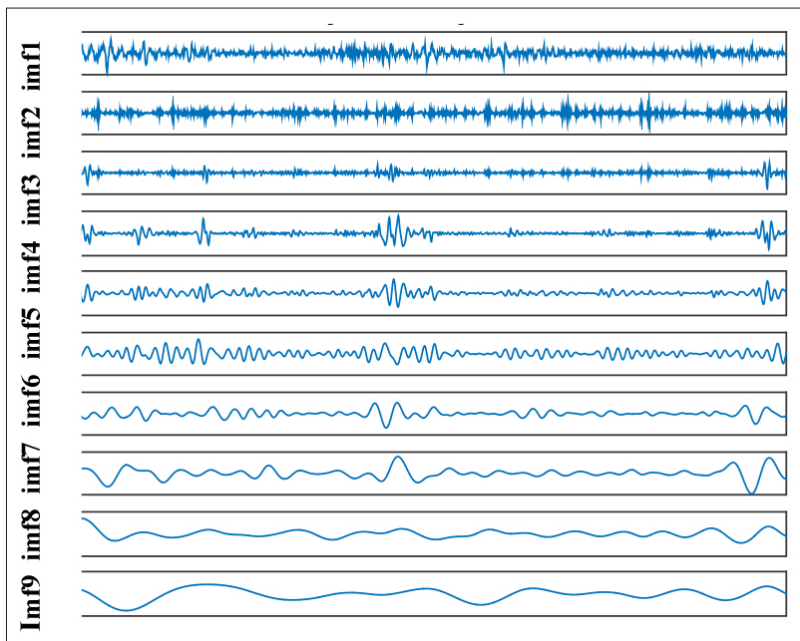

Figure 6. Expansions of EEG signal to IMF, using the differences between IMF oscillations of NA-MEMD and MEMD algorithms
Indeed, as can be seen in Figure 6, the difference began with the first IMF, due to NA-MEMD, and other inherent oscillations in the signal appeared. If looked at closely, new oscillations begin to emerge from the IMF 5 in Figure 5 as well as the easily noticed oscillations in Figure 6.

Accordingly, it is predicted that the NA-MEMD method would provide better results than the MEMD in emotional state classification studies.

Furthermore, to indicate the success of the MEMD method in filter bank structure, the well-defined instant frequencies for each IMF were shown and the mode-mixing state was presented in the channels used. Accordingly, as can be seen in Figure 7, the MEMD in terms of dyadic filter bank was successful, but more satisfactory results are obtainable. Additionally, considering EEG signals expanded to their IMFs, the oscillations are decreasing starting from the IMF 5 and almost no oscillations are observed in the IMF9.

Moreover, to show the success of the dyadic filter structure for the NA-MEMD used in the proposed emotion model, the mode-mixing state is demonstrated versus the well-defined instant frequencies for each IMF in the channels used in Figure 8. Accordingly, the dyadic filter bank structure in the NA-MEMD method was more successful than the MEMD dyadic filter bank when Figures 7, 8 were compared. It appeared that after IMF 5, the mode-mixing phenomenon diminished, and the mode alignment and oscillations increased.

Accordingly, a second definition for mode mixing phenomena may be evaluated depending on filter bank properties of NAMEMD and MEMD methods, demonstrated in Figure 7, 8 as:

$$
\begin{aligned}
& \operatorname{PSD}(I M F)_{M o d-M i x i n g}=\sum_{i=1}^{I} \sum_{j=1}^{J}\left(P S D_{N A-M E M D}(I M F)\right)_{i, j}- \\
& \sum_{i=1}^{I} \sum_{j=1}^{J}\left(P S D_{M E M D}(I M F)\right)_{i, j}
\end{aligned}
$$

Where " $i$ " stands for i.th indexed IMF for the modes $i=1,2,3, \ldots ., I$ and " $\mathrm{j}$ " stands for $\mathrm{j}$.th for the number of channel $j=1,2,3, \ldots . ., J$. With respect to the PSD, it represents the power spectral density in log scale.

As such, in this study our goal is to utilize these inherent features of the signal which appeared thanks to NA-MEMD method, as can be seen in Figure 4-8.

\section{Channel Selection and Operating MEMD and Algorithms}

Considering the EEG signals of the 40 videos of 32 participants in DEAP dataset utilized in the study, a total of 26 channel data for each participant were used. For each video viewing, 8000 data samples were selected. The 26 channels employed here were the channels on the left frontal-weighted lobe (Fp1, AF3, 


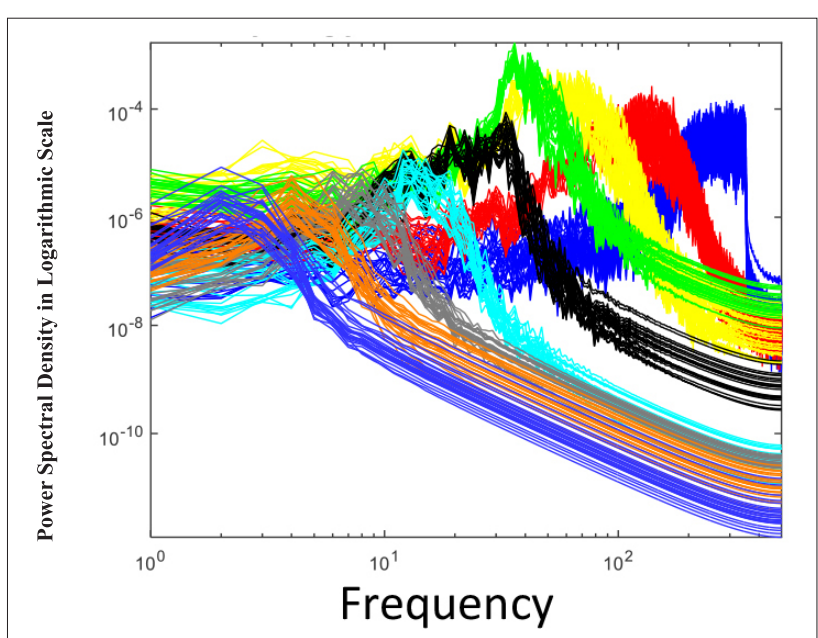

Figure 7. Mod mixing demonstration on IMFs using MEMD as a filter bank with using our all channels ( $V=128$ ) (10th participant's 20th video)

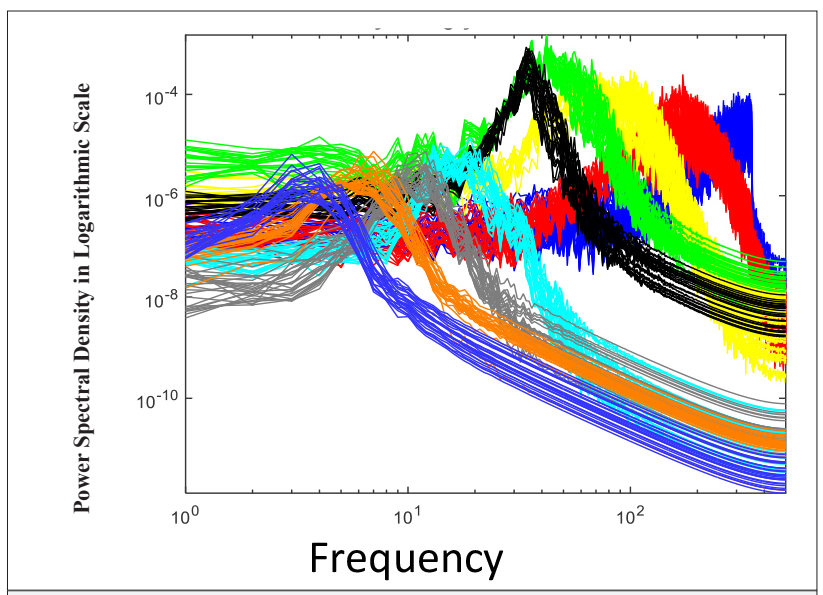

Figure 8. Mod mixing demonstration on IMFs using NA-MEMD as a filter bank with using our all channels $(V=128)$ (10th participant's 20th video)

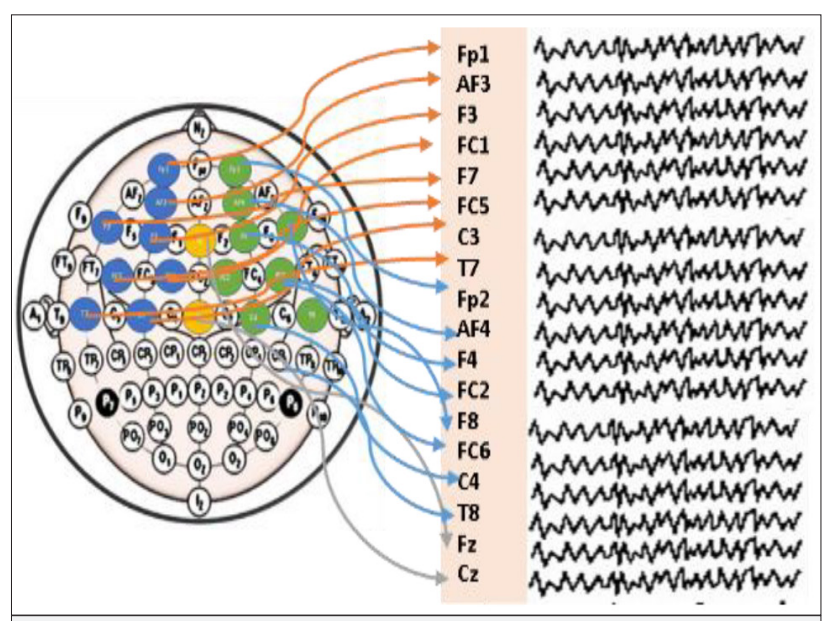

Figure 9. Representation of EEG electrode setting for the DEAP dataset
$\mathrm{F} 3, \mathrm{F7}, \mathrm{FC1}, \mathrm{FC5}, \mathrm{T7}, \mathrm{C} 3$ ), right frontal-weighted lobe (Fp2, AF4, $F 4, F 8, F C 2, F C 6, T 8, C 4)$, right and left frontal-weighted lobe differences, and 2 central channels ( $\mathrm{Fz}, \mathrm{Cz}$ ) CITATION Ahm $16 \mathrm{~V}$ 1055 [21].This data set is arranged as an input to the MEMD algorithm as can be seen in Figure 9. Thus, the obtained data set had a size of almost three-dimensional matrix as [26(channel) $\times 15$ (IMF number) $x 8000$ (data)]. Here, the center number demonstrates the number of IMFs. In this investigation, our IMF numbers varied between 13 and 17, and because the average mode number is 15 different video viewing sessions. The mode selected was 15 in our study.

Then, NA-MEMD method was executed. However, for this process, before the computation starts, the key issue was to pick a variance (power) of $2-10 \%$ of the variance of the input on the EEG signals recorded during every video of each participant. According to Algorithm 3 presented in section 3.4, $n=26$ (multivariable signal channel) and $q=2$ (number of channels used in noise addition) were prepared before the running process of the MEMD algorithm. As such, two noise channels corresponding to SNR ranging from 0.2 to $0.5 \mathrm{~dB}$ were employed in the noise subspace of and added to our " $n$ " channel. Hence, $(n+q)$ channels for data were acquired as $(26+2=28)$. The number of direction vectors in NA-MEMD was 128 and stopping criteria was $[\sigma 1=0.05, \sigma 2=0.5, \alpha=0.05]$. However, there is no restriction on the amount of noise channels to attach to the data set. NAMEMD method was executed for each video of each participant. In the content of $\mathrm{q}=2$ and $\mathrm{N}=8000$ (data length), independent noise sets were produced for WGN channels. Hereby, the obtained data was approximately three-dimensional matrix as [28 (channel) x15 (IMF number) x 8000(data)]. The number of IMFs do not affect the result due to the NA-MEMD calculation compared to MEMD results. The last two channels were eliminated due to the rule for the NA-MEMD method. Finally, the result as [(26 (channel)x 15 (IMF number) x8000(data)])] was obtained in the NA-MEMD method as well as in MEMD method.

\section{Classification}

Prior to the classification steps, the three-dimensional data set was converted into a one-dimensional data set. A total of 32 participants' dimensional feature sets were extracted utilizing the proposed NA-MEMD and MEMD method. At that point, the singular value decomposition (SVD) method was used for dimension reduction, which resulted in 1280 feature set, considering the first four IMF numbers [14]. For the classification steps, only the amplitudes of IMFs were applied compared to the reference in which further operations were executed after IMF computations [21]. After this process, for the data coming from 32 subjects, an input data was formed (each video of a participant was arranged as a row of sub-platforms), and the input matrix was composed. In terms of the output, for each label (valence-arousal-dominance) scale, it was resolved that the numbers ranging from 1 to 9 was changed as high and low labels. When the number was greater than 5 it was assigned as high, otherwise low. Then, with three different $1280 \times 1$ matrices 


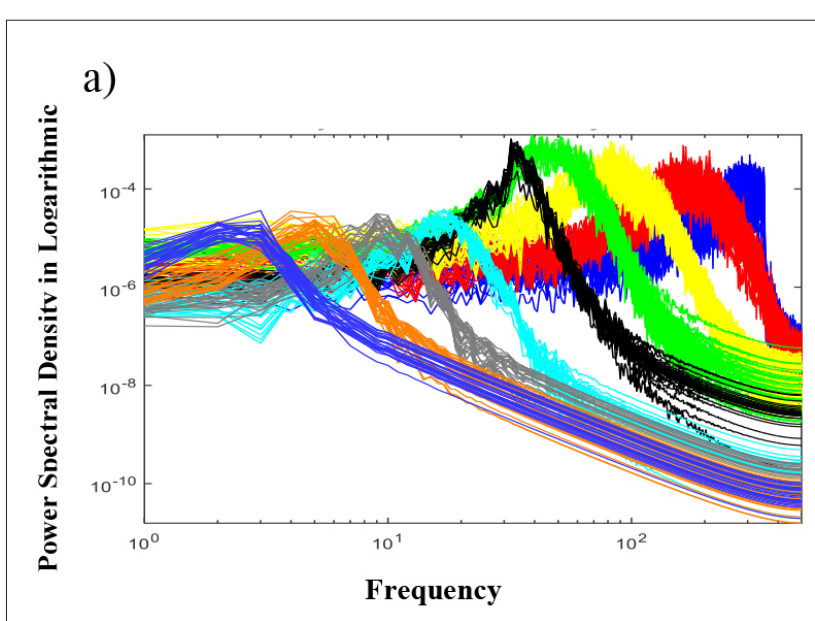

b)

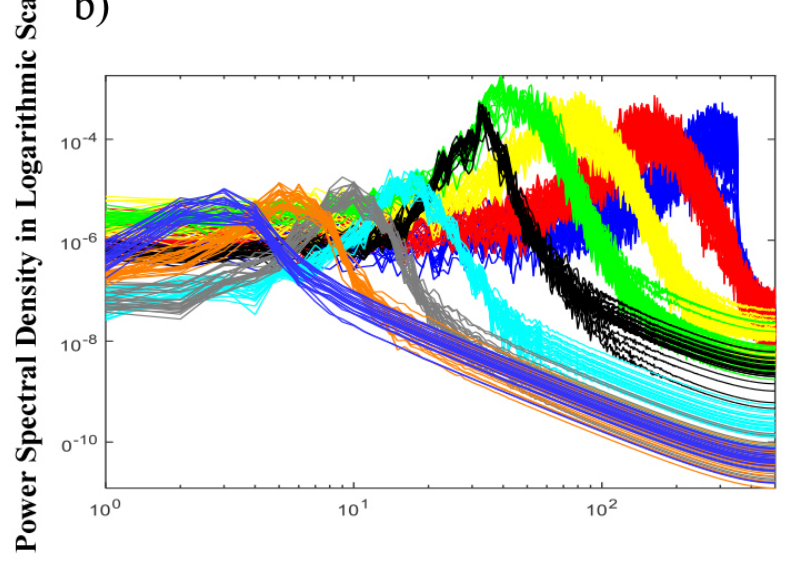

Frequency

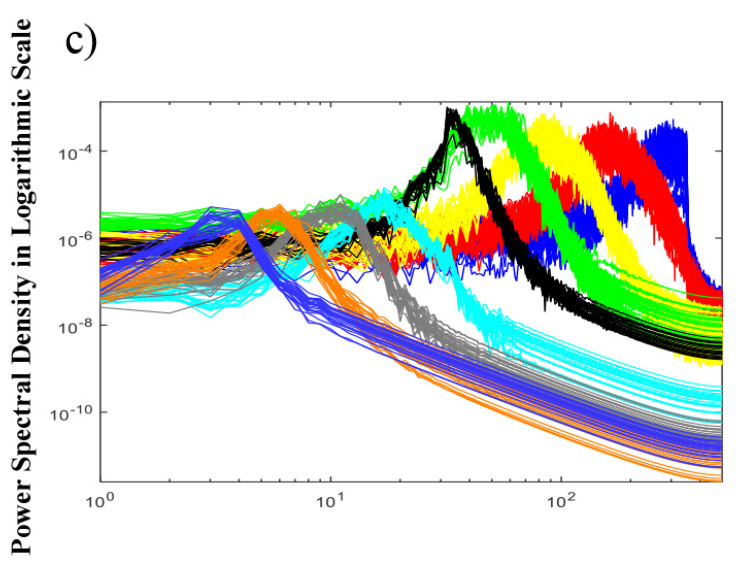

Frequency

Figure 10. a-c. IMFs alignment using NA-MEMD as a filter bank with using our all channels (a) $V=64$ b) $V=32$ c) $V=128)\left(12^{\text {th }}\right.$ participant's $10^{\text {th }}$ video.)

for valence-arousal-dominance scale, the execution of the selected classifier in Matlab Classification Learner Tool was calculated as $\{$ data $=$ [output (label data $(1280 \times 1)$ (e.g. valence label) input (processed EEG signal [1280x1]).

\section{Results}

In the proposed emotional state analysis model, band limited IMFs were obtained utilizing the dyadic filter bank structure that solves the mode-mixing problem of NA-MEMD in the way mentioned above. At the same time, the obtained IMFs' oscillations were used as the feature vector. As shown in Figure 9, instead of using the full 32 channels, the frontal weighted channels that cause emotional formation were used to obtain the effective result. The results are presented separately for comparison with MEMD and NA-MEMD. As previously mentioned, it is a prerequisite for NA-MEMD to select appropriate amplitudes (pick a variance (power) of $2-10 \%$ of the variance of the input) for the added noise channels to operate the algorithm successfully. The standard EMD appeared to be a special case of NAMEMD for very small infinite input noise levels. When the noise level was not in that range, it could lead to loss of the data-driven nature of NA-MEMD due to extreme input noise power [24]. The fact that the IMFs obtained by NA-MEMD were localized more frequently, and that there was a greater distinction between IMF frequency distributions, demonstrated a better consistency in the EEG components among the trials and showed that these elements were better separated. Meanwhile, IMF numbers resulting in the NA-MEMD algorithm was the same number as MEMD algorithm. When the filter bank structure and the IMF expansions were examined, the differences in the filter bank structure were apparent starting from IMF 5 (Figure 7, 8). Therefore, the classification results obtained by IMFs' contribution via MEMD, NA-MEMD algorithm using different classifiers are presented in Table 1.

Accordingly, the most satisfactory results belong to outcomes of NA-MEMD algorithm. So, via NA-MEMD, our accuracy rates were $70.0 \%, 73.8 \%$ and $74.2 \%$ respectively for high/low valence, arousal and dominance dimensions utilizing weighted k-NN Classifier. The second outstanding classification rates belong to Subspace k-NN Ensemble classifier utilizing NA-MEMD. And, the classification rates using Complex Decision Tree also have satisfactory results among the classifiers we used.

Those with the highest value for accuracy rate are among dominance dimensions. As shown in Table 1, almost all satisfactory results were obtained using the NA-MEMD method. Hence, it can be said that emerging the hidden inherent features in the IMFs utilizing NA-MEMD method positively contributes to the classification process.

\section{Conclusion}

The improvements provided by NA-MEMD showed that this method is a feasible alternative to MEMD when real emotional state is estimated from EEG data, due to reduced noise on IMF outputs, much more inherent signal features, and improved frequency localization. Noise-assisted methods, with the inclusion of both EEMD and NA-MEMD, will be helpful for signals with the dyadic filter bank decomposition. However, 
Table 1. Classification results obtained by IMFs' contribution via MEMD and NA-MEMD algorithm using Support Vector Machine, Decision Tree, k-Nearest Neighbor and Ensemble Classifier

\begin{tabular}{|c|c|c|c|c|}
\hline High-Low & Valence & Arousal & Dominance & Method \\
\hline Support Vector Machine (Fine Gaussian SVM) & $60.9 \%$ & $55.5 \%$ & $63.5 \%$ & MEMD \\
\hline Support Vector Machine (Fine Gaussian SVM) & $63.1 \%$ & $56.4 \%$ & $66.2 \%$ & NA-MEMD \\
\hline Decision Tree (Complex Tree) & $66.3 \%$ & $65.0 \%$ & $69.7 \%$ & MEMD \\
\hline Decision Tree (Complex Tree) & $68.4 \%$ & $67.2 \%$ & $71.9 \%$ & NA-MEMD \\
\hline k-Nearest Neighbor (Weighted k-NN) & $67.3 \%$ & $71.6 \%$ & $72.1 \%$ & MEMD \\
\hline k-Nearest Neighbor (Weighted k-NN) & $70.0 \%$ & $73.8 \%$ & $74.2 \%$ & NA-MEMD \\
\hline Ensemble (Subspace k-NN) & $66.4 \%$ & $69.6 \%$ & $70.0 \%$ & MEMD \\
\hline Ensemble (Subspace k-NN) & $68.1 \%$ & $72.3 \%$ & $72.5 \%$ & NA-MEMD \\
\hline
\end{tabular}

Table 2. The comparison of the NA-MEMD based emotion recognition with previous MEMD based studies

\begin{tabular}{|c|c|c|c|c|c|}
\hline Study & Dataset & $\begin{array}{l}\text { Emotion } \\
\text { Recognition }\end{array}$ & Accuracy Rates & Methods & Classifiers \\
\hline $\mathrm{Xu}$ & IAPS & $\begin{array}{l}\text { Positively excited, } \\
\text { Negatively excited, } \\
\text { Neutral }\end{array}$ & $\begin{array}{l}\text { MEMD-statistical; k-NN(81.39; LDA(59.18) } \\
\text { MEMD-NBP;k-NN (82.62);LDA(63.49) } \\
\text { MEMD-HOC;k-NN(90.77);LDA(79.64) } \\
\text { MEMD-Wavelet;k-NN(77.4);LDA(55,99) }\end{array}$ & $\begin{array}{l}\text { Memd-based } \\
\text { features, }\end{array}$ & $\begin{array}{l}\text { k-NN, } \\
\text { LDA }\end{array}$ \\
\hline Mert and Akan & DEAP & $\begin{array}{l}\text { High-Low Valence } \\
\text { High-Low Arousal } \\
\text { High-Low Valence } \\
\text { High-Low Arousal }\end{array}$ & 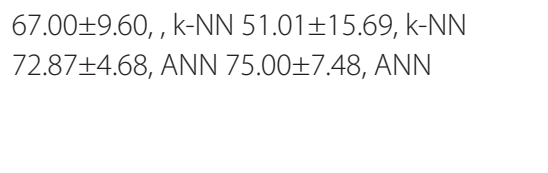 & $\begin{array}{l}\text { Memd-based features, } \\
\text { ICA, }\end{array}$ & $\begin{array}{l}\text { k-NN, } \\
\text { ANN }\end{array}$ \\
\hline Guitton & Self-Dataset & $\begin{array}{l}\text { Happy } \\
\text { Relaxed } \\
\text { Neutral } \\
\text { Fear }\end{array}$ & $\begin{array}{l}\text { MEMD,(SCA -FFA), SVM 72.2- } 83.377 .8-66.7 \\
72.2-83.366 .7-55.6\end{array}$ & Memd-based features, & $\begin{array}{l}\text { SVM, } \\
\text { k-NN } \\
\text { LDA }\end{array}$ \\
\hline
\end{tabular}

if the desired signal is in multiple sub-bands, selecting noisebased methods for analysis can "spread" the desired signal to multiple IMFs, causing undesirable mode mixing. In such cases, the implementation of a dyadic filter bank structure in the input signal can decrease the inherent data adaptive capability of EMD-based algorithms. Therefore, it is very crucial to choose the noise variance according to the input data. Our analysis also shows that the ideal noise levels for NA-MEMD are within $2-10 \%$ of the input data power (variance) [24]. This remark is represented in the simulations for the filter bank property belonging to the $10^{\text {th }}$ participant's $20^{\text {th }}$ video data (Figure 4-8) and the $12^{\text {th }}$ participant's $10^{\text {th }}$ video data (Figure $10,11)$ in DEAP dataset. The NA-MEMD algorithm gave better results than the MEMD algorithm in classification results, although there were some uncertainties. In the simulation results shown for the filter bank property in Figure 10, it is seen that choosing direction vector number 128 contributes to more meaningful IMF results. Optimal results were obtained by selecting both the number of direction vector 128 and using NA-MEMD. The disadvantage of large selection of the number of direction vector is that as the number of direction vector enhances, the time required to decompose the signal also enhances. In the IMF expansions obtained after analyzing EEG signals, it is important to note that the method is flexible, that the physically meaningful components of the analysis result are generated, and that the noisy noise power added to this data supervises the operation of the algorithm. NAMEMD's filter bank structures are illustrated in Figure 8, 10; in terms of noise interference and mode mixing, compared with the standard MEMD method. However, the disadvantage of the method is that after finding the variance of all the channels of EEG data the calculation of which is time consuming, adding the process of the variance noise ranging from $2-10 \%$ is computationally expensive and must be implemented with caution. To acquire a physically meaningful result from such data sets, all observed variables should be decomposed to the same number as the IMF components at similar scales. In such cases, NA-MEMD application provides great chan- 


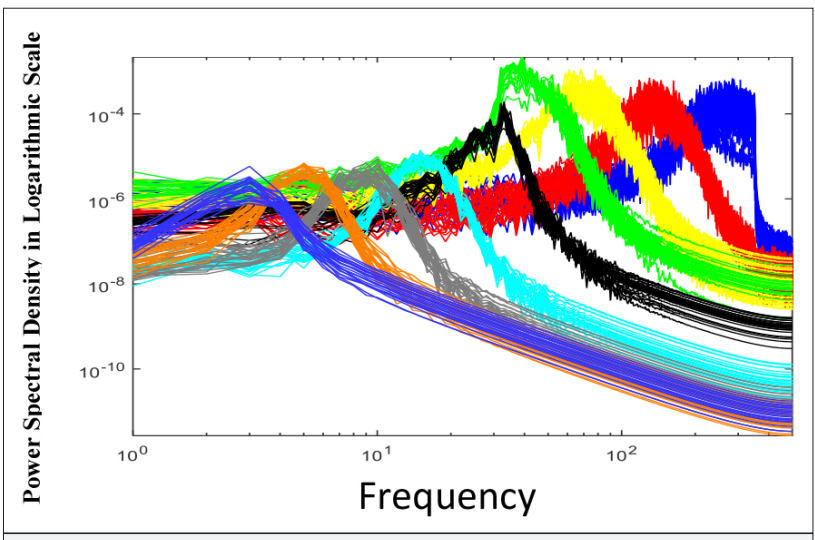

Figure 11. IMFs alignment using MEMD as a filter bank $(\mathrm{V}=128)$ (12 $2^{\text {th }}$ participant's $10^{\text {th }}$ video.)

nel-based advantages because it provides the same number of IMFs compared to MEMD. NA-MEMD method is better but the fact that NA-MEMD is preferred is directly related to the user's desire to work and to time planning. Nevertheless, the multivariable extensions of the EMD have the advantage of being more practical in handling the multichannel signals than EMD processing of each channel.

Considering MEMD based emotion recognition studies summarized in Table 2, our study is significant in that it uses embedded oscillations of MEMD within itself, evacuating via NAMEMD method. We acquired the best results $70.0 \%, 73.8 \%$ and $74.2 \%$ respectively for high/low valence, arousal and dominance (the mean rate was $72.6 \%$ ) dimensions utilizing weighted k-NN Classifier.

Study MEMD combined with Synchrony Cluster Analysis (SCA), Frequency Flows Analysis (FFA) and the spectrogram and obtain the maximum mean rate of $73 \%$ utilizing SCA calculated on the IMFs along with a SVM classifier [22]. Moreover, Mert and Akan take out Hjorth's parameters, entropy, power, PSD, correlation, and asymmetry power difference, power ratios, relative entropy, correlation, coherence of IMFs as features via MEMD and acquired the best results as 75.00 \pm 7.48 , ANN classification rates for high/low arousal. The presented method differs in that while our study uses and focuses on the inherent features during MEMD process. In addition, proposed their method based on MEMD however, they examine separately their feature extraction methods after obtaining IMFs [19, 22]. It is important to note that the choice of EEG channels, IMF selection, dimension reduction methods, the use of different datasets can cause different results.

The computation times necessary for the execution of the MEMD and NA-MEMD approaches with a personal computer were almost 8 , and 16 hours respectively for all video recordings of a participant. So, that utilizing NA-MEMD depends on the researcher's own preference even if the nature of data is appropriate for NA-MEMD method.
It should be noted that the multivariate signal processing approach is preferred in many applications. In this respect, EEG data are naturally multivariate and form an important link between different channels and multi-variate methods. As a result, for the analysis of multivariate data, the advantages of the NA-MEMD method over MEMD in the field of emotion-state classification are demonstrated using the fundamental issues of standard EMD - frequency localization, noise interference and mode-mixing. In addition, using EEG analysis using NAMEMD, due to the non-stationary and multichannel structure, the ability to explain the uncertainty over the methods using classic time frequency methods has been uncovered.

Peer-review: Externally peer-reviewed.

Conflict of Interest: The Authors have no conflicts of interest to declare.

Financial Disclosure: This study was supported by İmir Katip Çelebi University Scientific Research Projects Coordination Unit. Project number: 2017-ÖNAP-MÜMF-0002.

\section{References}

1. P. Ekman, W. V. Friesen, M. O'Sullivan, A. Chan, I. Diacoyanni-Tarlatzis, K. Heider, R. Krause, W. A. LeCompte, T. Pitcairn, P. E. Ricci-Bitti, "Universals and cultural differences in the judgments of facial expressions of emotion", J Pers Soc Psychol, vol. 53, no. 4, pp. 712-717, 1987

2. I. Bakker, T. V. der Voordt, P. Vink, J. de Boon, "Pleasure, Arousal, Dominance: Mehrabian revisited", Curr Psychol, vol 33, no. 3, pp. 405-421, 2014.

3. M. Othman, A. Wahab, I. Karim, M. A. Dzulkifli, I. F. T. Alshaiklia, “EEG emotion recognition based on the dimensional models of emotions", Procedia-Social and Behavioral Sciences, vol 97, pp. 30-37, 2013.

4. G. K. Verma, U. S. Tiwary, "Affect representation and recognition in 3D continuous valence-arousal-dominance space", Multimedya Tools Appl, vol. 76, no. 2, pp. 2159-2183, 2016.

5. Y. Liu, O. Sourina, "EEG Databases for Emotion Recognition", International Conference on Cyberworlds, 2013.

6. N. E. Huang, Z. Shen, S. R. Long, M. C. Wu,H. H. Shih, Q. Zheng, N. C. Yen, C. C. Tung, H. H. Liu, "The emprical mode decomposition and the Hilbert spectrum for nonlinear and non-stationary time series anaylsis", Proc Math Phys Eng Sci, vol. 454, NO. 1971, pp. 903-995, 1998.

7. N. E. Huang, Z. Wu, S. R. Long, K. C. Arnold, X. Chen, K. Blank, "On instantaneous frequency", Advanced Adaptive Data Anaysis, vol. 1, no. 2, pp. 177-229, 2009.

8. A. Khasnobish, S. Bhattacharyya, G. Singh, A. Jati, A. Konar, D. N. Tibarewala, R. Janarthanan, "The role of Emprical Mode Decompositıon on Emotion Classification Using Stimulated EEG signals", Advances in Computing and Information Technology, vol. 178, pp. 55-62, 2013.

9. A. Santillan-Guzman, M. Fischer, U. Heute, G. Schmidt, "Real-time Empirical Mode Decomposition for EEG signal enhancement", $21^{\text {st }}$ European Signal Processing Conference (EUSIPCO 2013), 2013.

10. P. C. Petrantonakis, L. J. Hadjileontiadis, "EEG-based emotion recognition using hybrid filtering and higher order crossings", $3^{\text {rd }}$ International Conference on Affective Computing and Intelligent Interaction and Workshops, 2009. 
11. P. C. Petrantonakis, L. J. Hadjileontiadis, "Emotion Recognition from Brain Signals Using Hybrid Adaptive Filtering and Higher Order Crossings Analysis", IEEE Transactions on Affective Computing, vol. 1, no. 2, pp. 81-97, 2010.

12. P. C. Petrantonakis, L. J. Hadjileontiadis, "Adaptive Emotional Information Retrieval From EEG Signals in the Time-Frequency Domain", IEEE Transactions on Signal Processing, vol. 60, NO. 5, pp. 2604-2616, 2012.

13. C. Shahnaz, S. B. Masud, S. M. S. Hasan, "Emotion recognition based on wavelet analysis of Empirical Mode Decomposed EEG signals responsive to music videos", IEEE Region 10 Conference (TENCON), 2016.

14. Y. Zhang, X. Ji, S. Zhang, " An approach to EEG-based emotion recognition using combined feature extraction method", Neurosci Lett, vol. 633, pp. 152-157, 2016.

15. M. S. Hosseini, A. Pouyan, S. Ferdowsi, H. Mashayekhi,"EEG-Based Emotion Recognition using Deep Belief Network and Empirical Mode Decomposition", $3^{\text {rd }}$ National Conference on Applied Research in Electrical, Mechanical and Mechatronic, 2015.

16. T. M. Rutkowski, A. Cichocki, A. L. Ralescu, D. P. Mandic, “Emotional States Estimation from Multichannel EEG Maps", Advances in Cognitive Neurodynamics ICCN 2007, 2008, pp. 695-698.

17. V. Bajaj, R. B. Pachori, "EEG Signal Classification Using Empirical Mode Decomposition and Support Vector Machine", International Conference on Soft Computing for Problem Solving, 2011.

18. P. Ozel, A. Akan, B. Yılmaz, "Emotional State Analysis from EEG signals via Noise-Assisted Multivariate Empirical Mode Decomposition Method", $10^{\text {th }}$ International Conference on Electrical and Electronics Engineering, 2017.

19. H. Xu, "Towards Automated Recognition of Human Emotion using EEG", Thesis, Toronto, Canada: University of Toronto, 2012.

20. Y. Tonoyan, D. Looney, D. P. Mandic, M. M. Van Hulle, "Discriminating Multiple Emotional States from EEG Using a Data-Adaptive, Multiscale Information-Theoretic Approach", Int J Neural Syst, vol. 26, no. 2, p. 1650005, 2016.

21. A. Mert, A.Akan, “Emotion recognition from EEG signals by using multivariate empirical mode decomposition", Pattern Analysis and Applications, vol. 21, no. 1, pp. 81-89, 2016.

22. C. Guitton, "Emotions Estimation from EEG Recordings", London: Imperial Collage of Science, Technology \& Medicine Department of Electrical\&Electronic Engineering, 2010.

23. H. T. Wu, P. Flandrin, I. Daubeschies, "One or two frequencies? The Synchrosqueezing Answers", Advances in Adaptive Data Anaylsis, vol. 3, no. 01n02, pp. 29-39, 2011.

24. N. U. Rehman, C. Park, N. E. Huang, D. P. Mandic, "Emd via Memd:Multivariate Noise Aided Computation of Standard Emd" Advances in Adaptive Data Analysis, vol. 5, no. 2, p. 1350007, 2013.

25. A. Ahrabian, D. Looney, F. A. Tobar, J. Hallatt, D. P. Mandic, “Noise Assisted Multivariate Empirical Mode Decomposition Applied to Doppler Radar Data", Sensor Signal Processing for Defence (SSPD 2012), 2012.

26. Q. S. She, Y. L. Ma, M. Meng, X. G. Xi, Z. Z. Luo, "Noise-assisted MEMD based relevant IMFs identification and EEG classification", Journal of Central South University, vol. 24, no. 3, p. 599-608, 2017.

27. J. Alegre-Cortés, S. Sánchez, A. G. Pizá, A. L. Albarracín, F. D. Farfán, C. J. Felice, E. Fernández, "Time-frequency analysis of neuronal populations with instantaneous resolution based on noise-assisted multivariate empirical mode decomposition", J Neuroscie Methods, vol. 267, pp. 35-44, 2016.

28. D. Cho, B. Min, J. Kim, B. Lee, "EEG-based Prediction of Epileptic Seizures Using Phase Synchronization Elicited from Noise-Assisted Multivariate Empirical Mode Decomposition", IEEE Transs Neural Syst Rehabil Eng, vol. 25, no. 8, pp. 1309-1318, 2017.

29. Lin,C.S, Tanumihardja, W.A., Shih,H.H., "Lung-heart sound separation using noise assisted multivariate empirical mode decomposition", International Symposium on Intelligent Signal Processing and Communication Systems, 2013.

30. Y. Zhang, P. Xu, P. Li, K. Duan, Y. Wen, Q. Yang, T. Zhang, D. Yao, "Noise Assisted Multivariate Empirical Mode Decomposition for Multichannel EMG Signals," BioMedical Engineering Online, vol. 16, no. 107, pp. 1-17, 2017.

31. S. Sungkono, B. J. Santosa, A. S. Bahri, F. M. Santos, A. Iswahyudi, "Application of Noise Assisted Multivariate Empirical Mode Decomposition in VLF-EM Data to Identify Underground River", Advances in Data Science and Adaptive Analysis, vol. 9, no. 1, p. $1650011,2017$.

32. W. Huang, J. Zeng, Z. Wang, J. Liang, "Partial noise assisted multivariate EMD:An improved noise assisted method for multivariate signals decomposition", Biomedical Signal Processing and Control, vol. 36, pp. 205-220, 2017.

33. S. Koelstra, C. Muhl, M. Soleymani, J. S. Lee, A. Yazdani, T. Ebrahimi, T. Pun, A. Nijholt, I. Patras, "DEAP: A database for emotion analysis; using physiological signals", IEEE transactions on affective computing, vol. 3, no. 1, pp. 18-31, 2012.

34. N. Rehman, D. P. Mandic, "Multivariate empirical mode decomposition", Proc Math Phys Eng Sci, vol. 466, no. 2117, pp. 1291-1302, 2009.

35. P. Flandrin, P. Gonçalves, G. Rilling, "EMD equivalent filter banks, from interpetation", Hilbert-Huang Transform and Its Applications, Singapore, World Scientific, 2005, pp. 67-87.

36. Z. Wu, N. E. Huang, "Ensemble Emprical Mode Decomposition : A Noise Assisted Data Analysis Method", Advances in Adaptive Data Analysis, vol. 1, no. 1, pp. 1-41, 2009.

37. M. E. Torres, M. A. Colominas, G. Schlotthauer, P. Flandrin, "A complete ensemble empirical mode decomposition with adaptive noise", IEEE International Conference on Acoustics, Speech and Signal Processing (ICASSP) Prague, 2011.

38. J. R. Yeh, J. S. Shieh, N. E. Huang, "Complementary ensemble empirical mode", Advanced Adaptive Data Anaylsis, vol. 2, no. 2, pp. 135-156, 2010.

39. Hao, H., Wang, H., Rehman, N.U., Tian, H., "A Study of the Characteristics of MEMD for Fractional Gaussian Noise" IEICE Transactions on Fundamentals of Electronics, Communications and Computer Sciences, vol E99.A, no. 6, pp. 1228-1232, 2016.

40. S. Ge, Y. H. Shi, R. M. Wang, P. Lin, J. F. Gao, G. P. Sun, K. Iramina, Y. K. Yang, Y. Leng, H. Wang, W. Zheng, "Sinusoidal Signal Assisted Multivariate Empirical Mode Decomposition for Brain-Computer Interfaces", IEEE Journal of Biomedical and Health Informatics, 2017.

41. N. ur Rehman, D. P. Mandic, "Filter bank property of multivariate empirical mode decomposition", IEEE transactions on signal processing, vol. 59, no. 5 , pp. 2421-2426., 2011. 
PInar Özel received her BS degree in 2007 in Erciyes University and MS degree in 2011 from Bogazici University. She started her PhD studies in 2013 in Istanbul University and she's continuing her studies. As professional experience, she worked as a product manager. Her research areas are signal processing, techniques to biomedical signals.
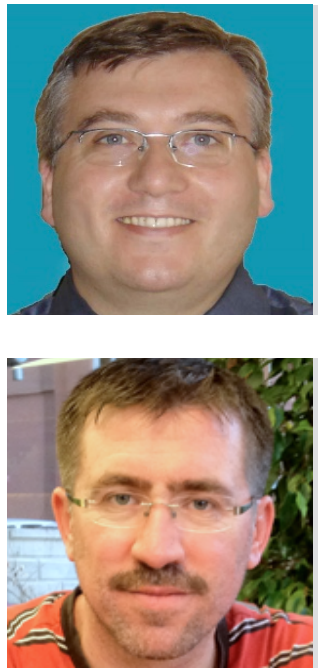

Aydın Akan received the B.Sc. degree from the University of Uludag, Bursa, in 1988, the M.Sc. degree from the Technical University of Istanbul, Istanbul, Turkey in 1991, and the Ph.D. degree from the University of Pittsburgh, Pittsburgh, PA, USA, in 1996, all in electrical engineering. He was with the Department of Electrical and Electronics Engineering, University of Istanbul, between 1996 and 2017. He is currently a Professor at the Department of Biomedical Engineering, Izmir Katip Celebi University, Izmir, Turkey. His current research interests include nonstationary signal processing, time-frequency signal analysis methods and their applications to wireless communications and biomedical engineering. He is a senior member of the IEEE Signal Processing Society and an Associate Editor of the Digital Signal Processing Journal.

Bülent Yılmaz received his BS and MS degrees in Electrical-Electronics Engineering from the Middle East Technical University, Ankara, in 1997 and 1999. He obtained his PhD degree from the Bioengineering Department of the University of Utah, Salt Lake City, Utah. He has been working as a faculty member since 2011 in Abdullah Gül University, Department of Electrical-Electronics Engineering, Kayseri. His current research interests include biomedical signal and image processing applications on brain-computer interfaces, texture analysis on colonoscopic and PET/CT images. 